\title{
The Second Special Issue of The Horticulture Journal—Strawberry-
}

\section{Masayoshi Shigyo $^{1,2}$ and Takeshi Kurokura ${ }^{1,3}$}

\author{
${ }^{1}$ Editorial office, The Horticulture Journal (Hort. J.) \\ ${ }^{2}$ Yamaguchi University, Yamaguchi 753-8515, Japan \\ ${ }^{3}$ Utsunomiya University, Utsunomiya 321-8505, Japan
}

The Japanese Society for Horticultural Science, which publishes Hort. J., has organized this special issue to further enhance the attractiveness of this journal. The theme of the second special issue is "Strawberry" which include many aspects, from physiology and genetics of the genus Fragaria, breeding, production technique, postharvest physiology, and even to transportation and consumption.

Strawberry (Fragaria $\times$ ananassa Duch.) has become one of the commodity crops in East Asia so that the strawberry industry of this region (China, Japan, Korea, and Taiwan) has experienced an upward trend in yield as well as production area over the last decade. This is due to several reasons: consumers' awareness of the importance of healthy diet; yield improvements which evoked an expanded intraregional supply; novel cropping system together with unique varieties to allow year-round fruits production.

In Japan, a usual harvest period starts from late November and last in June with single cropping, and more than $90 \%$ is produced by forcing culture. The accumulation of the technological research and development for forcing contributes to such a long-term harvest. Considering that such technological factors for Japanese unique cropping system should be useful in the expansion of global strawberry production in the future, we planned this second special issue of Hort. J. 